Preprint typeset using $\mathrm{LAT}_{\mathrm{E}} \mathrm{X}$ style emulateapj v. 5/2/11

\title{
UP TO 100000 RELIABLE STRONG GRAVITATIONAL LENSES IN FUTURE DARK ENERGY EXPERIMENTS
}

\author{
S. SERJEANT \\ Department of Physical Sciences, The Open University, Milton Keynes, MK7 6AA, UK
}

\begin{abstract}
The Euclid space telescope will observe $\sim 10^{5}$ strong galaxy-galaxy gravitational lens events in its wide field imaging survey over around half the sky, but identifying the gravitational lenses from their observed morphologies requires solving the difficult problem of reliably separating the lensed sources from contaminant populations, such as tidal tails, as well as presenting challenges for spectroscopic follow-up redshift campaigns. Here I present alternative selection techniques for strong gravitational lenses in both Euclid and the Square Kilometer Array, exploiting the strong magnification bias present in the steep end of the $\mathrm{H} \alpha$ luminosity function and HI mass function. Around $10^{3}$ strong lensing events are detectable with this method in the Euclid wide survey. While only $\sim 1 \%$ of the total haul of Euclid lenses, this sample has $\sim 100 \%$ reliability, known source redshifts, high signal-to-noise and a magnification-based selection independent of assumptions of lens morphology. With the proposed Square Kilometer Array dark energy survey, the numbers of reliable strong gravitational lenses with source redshifts can reach $10^{5}$.

Subject headings: cosmology: observations — galaxies: evolution — galaxies: formation — galaxies: starburst - infrared: galaxies — submillimeter: galaxies
\end{abstract}

\section{INTRODUCTION}

The next generation of optical/near-infrared survey space telescopes, such as the Euclid $15000 \mathrm{deg}^{2}$ wide survey and the WFIRST $2000 \mathrm{deg}^{2}$ high latitude survey (Laureijs et al. 2011, Spergel et al. 2013), aim to make pioneering constraints on the evolving equation of state of dark energy. Their slitless $\mathrm{H} \alpha$ spectroscopy surveys use Baryonic Acoustic Oscillations to provide a cosmological standard ruler while the imaging surveys provide weak lensing constraints on dark energy. The full Square Kilometer Array (SKA) has also been proposed to be used to make comparable constraints on dark energy (e.g. Abdalla et al. 2010), through a hemispheric Hi redshift survey. In the process, every one of these dark energy experiments will also create huge legacy data sets that will benefit many areas of astronomy: e.g. $5 \sigma$ spectroscopic depths of $4.3 \times 10^{-16} \mathrm{ergs} \mathrm{cm}^{-1} \mathrm{~s}^{-1}$ and $0.36 \times 10^{-16}$ ergs cm${ }^{-1} \mathrm{~s}^{-1}$ for Euclid and WFIRST respectively, and $5 \sigma$ point source depths of $Y=J=H=24$ for Euclid and $Y=H=26.7, J=26.9$ for WFIRST.

Strong gravitational lensing particularly benefits from Euclid's $1.2 \mathrm{~m}$ diffraction-limited optical/near-infrared imaging over around half the sky, with $\sim 10^{5}$ galaxygalaxy lensing events predicted in the $15000 \mathrm{deg}^{2}$ wide survey (Laureijs et al. 2011). This is many orders of magnitude of improvement in the numbers of lenses. Many applications of strong gravitational lensing are limited by sample size (e.g. Treu 2010), such as dark matter halo substructure (e.g. Dalal \& Kochanek 2002), halo density profiles (e.g. Gavazzi et al. 2007, 2008), cosmological parameters (e.g. Short et al. 2012, Gavazzi et al. 2008, but see Schneider 2014), and extreme high magnification systems (e.g. Wang \& Turner 1996).

However, identifying these systems with high reliability and completeness is not without its difficulties. In a heroic effort, Jackson (2008) visually examined 285423 galaxies with $I<25$ in the Cosmic Evolution Survey
(COSMOS). He found two certain new lenses, one probable candidate and a further 112 candidates, to add to the then-known 20 COSMOS lenses and 47 candidates. False positives could be due, for example, to chance lensing-like locations of HII regions in a galaxy, or tidal tails resembling lensed arcs. Visual inspection of larger data sets is a much more challenging proposition. This problem has stimulated both the development of arc-finder algorithms in multi-wavelength imaging data (e.g. Gavazzi et al. 2014) and the creation the Spacewarps mass participation citizen science experiment].

In this paper I present alternative methods of identifying strong gravitational lens events, by exploiting the strong magnification bias in the high-luminosity end of the $\mathrm{H} \alpha$ luminosity function and the high-mass end of the HI mass function. This paper follows the unfashionably conventional format of introduction, method, results, discussion and conclusions in sections 1, 2, 3, 4 and 5 respectively. This paper assumes a Hubble constant of $H_{0}=70 \mathrm{~km} \mathrm{~s}^{-1} \mathrm{Mpc}^{-1}=100 h \mathrm{~km} \mathrm{~s}^{-1} \mathrm{Mpc}^{-1}$ and density parameters of $\Omega_{\mathrm{M}}=0.3$ and $\Omega_{\Lambda}=0.7$ throughout.

\section{METHOD}

In the presence of very steep number counts or luminosity functions, a strong magnification bias can operate. The low probability of galaxy-galaxy lensing producing an apparently-bright lensed galaxy can be more than compensated for by the presence of a very large population of faint candidate background lensed sources. If the slope of the differential numbers steepens at progressively brighter fluxes or luminosities, then the fraction of lensed sources also progressively increases.

This method of selecting strong gravitational lenses has a successful track record. Negrello et al. (2010) used this technique to select a sample of strong lenses using simply a $500 \mu \mathrm{m}$ flux limit of $S_{500 \mu \mathrm{m}} \geq 100 \mathrm{mJy}$, exploiting

\footnotetext{
1 http://spacewarps.org
} 

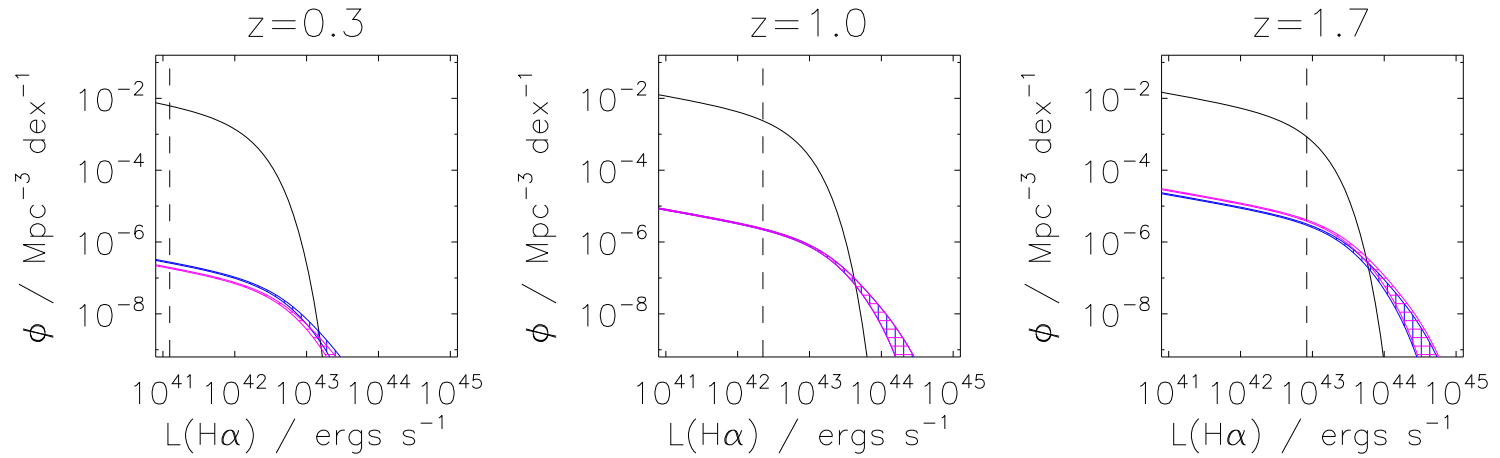

Figure 1. H $\alpha$ luminosity function from Geach et al. 2010 (black line) at redshifts $z=0.3$, 1.0 and 1.7, the singular isothermal sphere lenses of Perrotta et al. 2002 and Perrotta et al. 2003 (pink), and with a non-evolving population of lenses normalised to the Perrotta predictions at an arbitrarily chosen $z=1$. The hatched regions span the range of maximum magnification $\mu \leq 10$ and $\mu \leq 30$. The vertical lines show the fiducial $5 \sigma$ flux limit of the Euclid wide-field survey (Laureijs et al. 2011). Note that at $>12 \bar{L}{ }_{*}$ the observed population is dominated entirely by strong gravitational lens systems.
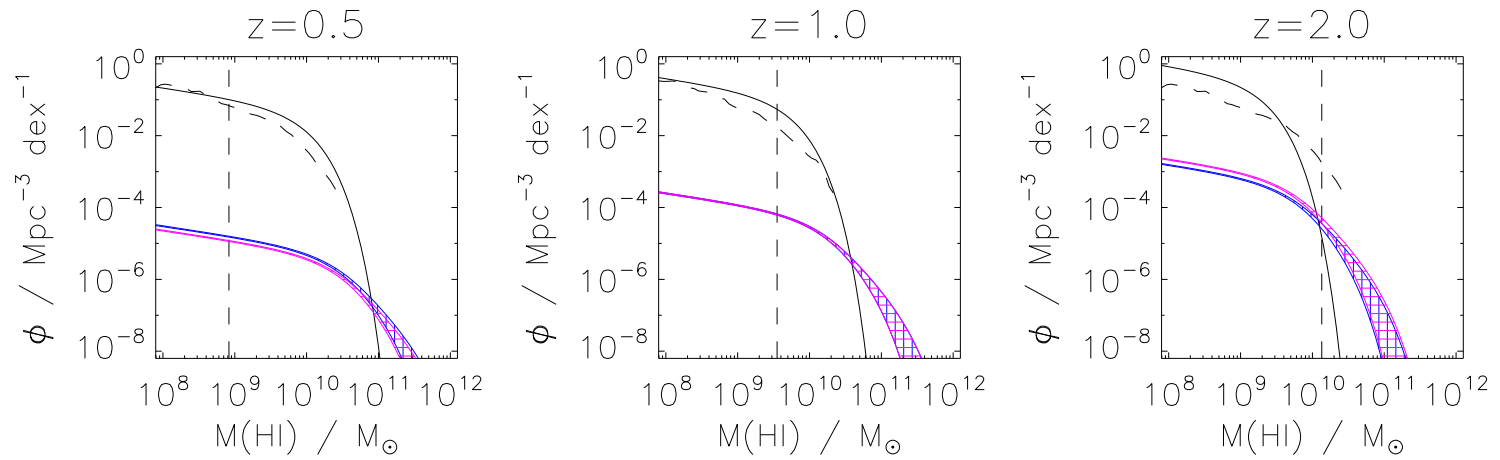

Figure 2. Hi mass function following Abdalla et al. (2010) model "C" at redshifts of $z=0.5,1.0$ and 2.0. Lensing models as in Fig.1 The vertical lines show the fiducial flux limit of the proposed full $S K A$ dark energy survey from Abdalla et al. (2010), assuming a sensitivity parameter $f=1$ (see Abdalla et al. 2010 equation 2), $30 \mathrm{~km} \mathrm{~s}^{-1}$ channels and a $100 \mathrm{deg}^{2}$ field of view. The dashed curve shows the evolving semi-analytic predictions of Lagos et al. (2011), for comparison. Similarly to Fig.1 note that at $>12 M_{*}$ the observed population is dominated entirely by strong gravitational lens systems.

the steep slope of submm number counts. Half of the selection were contaminant (non-lensed) local galaxies and blazars, but these were easily identifiable in supplementary optical and radio survey data, leaving a sample with an ostensible $\sim 100 \%$ reliability that the authors confirmed with a wide range of spectroscopic and imaging follow-ups (see also e.g. Viera et al. 2013, Bussmann et al. 2013). González-Nuevo et al. (2012) extended this methodology to the high-luminosity end of the submm luminosity function (estimated using submm photometric redshifts), and in the process expanded the number of gravitational lens candidates in Herschel surveys from $\sim 100$ with a bright flux limit to $\sim 1000$ with a bright luminosity limit in the Herschel $A T L A S$ project (Eales et al. 2010). After removing known contaminants the reliability of this strong gravitational lens sample was estimated to be $\sim 70 \%$, sufficient to warrant extensive follow-up campaigns (e.g. Amber et al. in prep.) that could yield constraints on cosmological parameters (e.g. Short et al. 2012), constraints on the evolution of dark matter halo properties, and/or constraints on the background lensed galaxy population (e.g. Eales 2014).

The $\mathrm{H} \alpha$ and far-infrared luminosities of star-forming galaxies have in common that they are dominated by the luminous output of massive stars, which excites nebular emission in HII regions in the case of $\mathrm{H} \alpha$, and which is absorbed by dust and re-emitted as grey-body radiation in the case of the far-infrared (e.g. Condon 1992 et seq.). Both $\mathrm{H} \alpha$ and far-infrared luminosities are widely used as star formation rate estimators, among other indicators. However, the $\mathrm{H} \alpha$ and far-infrared luminosities have different dependencies on star formation rate: at high star formation rates, a greater proportion of star formation is present in giant molecular cloud complexes, so the $\mathrm{H} \alpha$ luminosity will tend to plateau as star formation rate increases while the far-infrared continues to increase (e.g. Flores et al. 2004, Swinbank et al. 2004, Calzetti et al. 2010). Balmer lines are inevitably dominated by lowextinction regions (e.g. Serjeant et al. 2002), so even Balmer decrement-corrected $\mathrm{H} \alpha$ luminosities will underpredict the total star formation rate in the most extreme starbursts. Unless the extinction is accounted for empirically (e.g. Calzetti et al. 2010), naive interpretations of $\mathrm{H} \alpha$ luminosities consistently under-predict star formation rates for the most luminous starbursts at all redshifts (e.g. Sedgwick et al. 2013). For example, the $\mathrm{H} \alpha$ luminosities of hyperluminous infrared galaxies $\left(>10^{13} L_{\odot}\right)$ are not $\sim 100 \times$ brighter than those of luminous infrared 
galaxies $\left(>10^{11} L_{\odot}\right)$. Therefore, extreme $\mathrm{H} \alpha$-emitters should be rarer than extreme far-infrared emitters.

For this reason, it is reasonable to expect the $\mathrm{H} \alpha$ luminosity function to have a bright-end slope that is steeper than its far-infrared counterpart (e.g. Lapi et al. 2011, Marchetti et al. in prep.). The latter has already been demonstrated to facilitate strong gravitational lens selection (e.g. González-Nuevo et al. 2012), so one would expect the bright end of the $\mathrm{H} \alpha$ luminosity function to be similarly useful. This paper adopts the $\mathrm{H} \alpha$ luminosity function determination of Geach et al. (2010) at redshifts $0<z<2$, which is characterised by a Schechter function. Note that this determination includes both star-forming galaxies and active galaxies.

Another population statistic where theoretical models and (to some extent) observations lead to steep number counts and an expectation of strong magnification bias is the neutral hydrogen galaxy mass function. The physical processes determining the neutral hydrogen distribution are arguably simpler than the physics of star formation and dust obscuration. The local Hi mass function is welldescribed by a Schechter function (Zwaan et al. 2003), in agreement with the expectations from semi-analytic models (e.g. Baugh et al. 2004). At higher redshifts there are few empirical constraints, though Abdalla et al. (2010) used damped Lyman $\alpha$ system number densities and the evolving volume-averaged star formation history to determine the evolution of the break in the mass function, resulting in their preferred model, denoted model "C". The semi-analytic predictions of Lagos et al. (2011) evolve in the same sense as Abdalla's model "C" but the evolution is less pronounced. Therefore, in order to span the range of plausible predictions, this paper adopts the model "C" of Abdalla et al. (2010) and a conservative no-evolution model.

The formalism for calculating the differential magnification probability distribution $p(\mu, z) \mathrm{d} \mu$ for a magnification $\mu$ is summarised in Blain (1996), Perrotta et al. (2002) and Perrotta et al. (2003). Regardless of the lens population, the high magnification tail has the form $p(\mu, z)=a(z) \mu^{-3}$ for some function $a(z)$. For the purposes of this paper, "strong" lensing is taken to mean magnifications $\mu \geq 2$, in which this expression applies.

The form of $a(z)$ depends on the nature and evolving number density of lenses. Both Blain (1996) and Perrotta et al. (2002, 2003) consider several options for galaxy lens populations. In the case of Perrotta et al. (2002, 2003), the mass spectrum follows the Sheth and Tormen (1999) formalism. There is good evidence for the galaxy lens population being well-described by singular isothermal ellipsoids (e.g. Gavazzi et al. 2007, 2008) as opposed to Navarro Frenk White (1997) profiles, so this paper assumes a singular isothermal sphere profiles for the Perrotta et al. lenses. For an alternative approach, one can assume the mass spectrum of lensing galaxies does not evolve with redshift (following e.g. the approach of model "B" of Blain 1996, though with a different cosmology), and normalise $a(z)$ to the prediction of Perrotta et al. $(2002,2003)$ at an arbitrary redshift of $z=1$. These options should span the parameter space of plausible lens populations.

A more significant source of uncertainty in the lensing predictions is the maximum magnification imposed by the finite source sizes. This paper follows Perrotta et al. (2002) by spanning the range of plausible maximum magnifications with $\mu \leq 10$ and $\mu \leq 30$, as appropriate for source characteristic radii of $\sim 1-10 h^{-1} \mathrm{kpc}$ (e.g. Wuyts et al. 2013). High magnification events are known in far-infrared and submm-selected lenses (e.g. Swinbank et al. 2010), but this paper conservatively neglects this extreme population. The surface density of strong gravitational lenses increases with the maximum magnification; the choice in this paper is more conservative than that of e.g. Blain (1996) who used $\mu \leq 40$, and Lima et al. (2010) who used $\mu \leq 100$.

\section{RESULTS}

Fig.1 shows the effect of applying the strong lensing formalism discussed above to the Geach et al. (2010) $\mathrm{H} \alpha$ luminosity function. It is clear that at apparent luminosities $>10^{44} \mathrm{ergs} \mathrm{s}^{-1}$, the observed population is entirely dominated by strong gravitational lens systems, regardless of the maximum magnification or the nature of the lensing population. A redshift-dependent threshold based on the shape of the evolving unlensed luminosity function would yield a larger sample of lensed galaxies. This paper adopts $>12 L_{*}$ as a conservative fiducial limit for selecting a sample of strongly lensed $\mathrm{H} \alpha$ emission line galaxies. These samples have $\sim 97-99 \%$ reliablility, i.e. the unlensed contaminants are $1-3 \%$ of the total sample selected in this way. The fiducial $\mathrm{H} \alpha 5 \sigma$ flux limit for the Euclid wide-area survey of $4.3 \times 10^{-16} \mathrm{ergs} \mathrm{s}^{-1} \mathrm{~cm}^{-2}$ (Laureijs et al. 2011) is indicated as a vertical line in Fig.11 the strong lensing systems will be detected at very high signal-to-noise.

Assuming a fiducial sky coverage of $15000 \mathrm{deg}^{2}$ for the wide-area Euclid mission survey, the numbers of stronglylensed galaxies with luminosities $>12 L_{*}$ range from 1079 using the no-evolution model and $\mu \leq 10$ to 2717 with the Perrotta et al. $(2002,2003)$ model and $\mu \leq 30$.

An even larger haul of lenses is possible with the SKA HI surveys. With the HI mass limit estimated by Abdalla et al. (2010) for the fiducial one-year dark energy survey with the full SKA, and assuming a survey area of $15000 \mathrm{deg}^{2}$, the $>12 M_{*}$ limit yields of the order $10^{4}$ strong gravitational lenses with $96-98 \%$ reliability. The predictions range from 5800 lenses with the no-evolution lens population and $\mu \leq 10$ to 14000 with the Perrotta et al. (2002, 2003) model and $\mu \leq 30$. The surface density of strong lenses is higher than the Euclid $\mathrm{H} \alpha$ case, because the mass function Schechter normalisation is $\sim 10 \times$ higher than that that of the $\mathrm{H} \alpha$ Schechter function.

Model predictions of the evolution of the Hi mass function tend to dismantle local high-mass systems into a larger number of lower-mass systems at high redshift. This moves the characteristic mass scale $M_{*}$ to lower masses at high redshifts, and the normalisation $\phi_{*}$ to higher number densities. The effect on the lensing predictions is to greatly increase the strength of the magnification bias and the numbers of strong lensing systems. Figure 2 2 shows the predictions for the evolving mass function models. The SKA dark energy survey results in an astonishingly high surface density of lenses with $97-99 \%$ reliability, of the order $10^{5}$ in a $15000 \mathrm{deg}^{-2}$ survey: predictions range from 7400 lenses with the unevolving lens population and $\mu \leq 10$, to 190000 lenses with $\mu \leq 30$ and the Perotta models. The Lagos et al. (2011) models are 
in fairly good agreement with the Abdalla et al. (2010) predictions at $z<1$, but are discrepant at $z=2$ (Fig.(2), though still following a Schechter shape. In the Lagos case, the $>12 M_{*}$ threshold will be brighter than the Abdalla model, with high-redshift lensing numbers intermediate between the Abdalla and no-evolution cases.

\section{DISCUSSION}

Are the $\mathrm{H} \alpha$ emission lines in the lensing systems detectable by Euclid against the glare of the foreground lens? A simple calculation shows that this is typically not likely to be a problem. The median $H$-band magnitude of the SLACS lenses is $H=15.9$ (Auger et al. 2009), equivalent to a flux density of $4.7 \times 10^{-16} \mathrm{~W} \mathrm{~m}^{-2} \mu \mathrm{m}^{-1}$. At $z=1.5$, the $>12 L_{*}$ selection is equivalent to a flux limit of $5.7 \times 10^{-18} \mathrm{~W} \mathrm{~m}^{-2}$, approximately a factor of ten above the point source flux limit (Laureijs et al. 2011). With the reasonable assumption of an unresolved line at the expected resolving power of $\lambda / \Delta \lambda=500$, the observed line flux density would be around four times the continuum level from the lens. This is conservatively assuming the lensing galaxy is not extended, which would dilute the flux density contribution from the lens. This is also assuming that the $\mathrm{H} \alpha$ emission is also unresolved. The $3 \mathrm{D}-\mathrm{HST}$ project has found effective $\mathrm{H} \alpha$ radii at $z \sim 1$ of $\sim 2-4 \mathrm{kpc}$ (Wuyts et al. 2013), equivalent to $0.25-0.5$ arcseconds, small compared to the NISP spectrometer pixel scale of 0.5 arcseconds. With angular magnification from lensing, we may expect the $\mathrm{H} \alpha$ emission to be typically no more than marginally resolved by Euclid, apart from e.g. quad lens configurations.

The high surface density of strong galaxy-galaxy gravitational lens events in the proposed one-year SKA weak lensing survey may have a significant effect on the shear statistics. The strongly lensed population is identifiable at high Hi masses, but further work is needed to quantify the effect on the shear statistics of strongly lensed galaxies at lower Hi masses. However, as a resource in themselves, the HI-selected strong lensing systems are potentially revolutionary. There are no obvious significant unlensed contaminant populations for the HI-selected samples, but dedicated lensing Hi surveys with $S K A$ pathfinders/precursors would identify any such populations.

Having prior knowledge of the background source redshifts partly solves one of the major practical problems with strong gravitational lens surveys: the multiwavelength follow-ups. For example, most COSMOS gravitational lens systems still lack a secure measurement of the background redshift (e.g. Faure et al. 2011), including all three lens candidates from Jackson (2008). This problem is intrinsic to morphologicallyselected samples of lensing systems and will be a major challenge for morphologically-selected strong lenses from Euclid and WFIRST.

The $\mathrm{H} \alpha$ and HI-selected systems will nonetheless need redshift estimation of the foreground lenses. The lensing optical depth is maximised approximately half-way between the source and observer, so lens redshifts will be typically in the range $z \simeq 0.5-1$. Optical and nearinfrared ground-based photometric surveys such as the Large Synoptic Survey Telescope will be well-suited to obtaining photometric redshifts of massive galaxies at these redshifts. Spectroscopic absorption line lens red- shifts are likely to require $\sim 1$ hour exposures on $4 \mathrm{~m}$-class optical telescopes (e.g. Bussmann et al. 2013, Amber et al. in prep.). For early-type lenses selected with Euclid and WFIRST, additional lens redshift estimation will be possible from the fundamental plane (e.g. Serjeant et al. 1995, Eisenhardt et al. 1996). The design of the Sloan Lens ACS Survey (SLACS) means it is inevitably dominated by early-type lenses (e.g. Auger et al. 2009), but it is not at all clear that early-type lenses are necessarily responsible for nearly all the lensing optical depth in the Universe. By exploiting magnification bias, the $\mathrm{H} \alpha$ and HI selection methods are identifying high magnification lines of sight, irrespective of the nature of the foreground lenses. Submm samples are similarly selected on magnification bias, and as well as early-type lenses they include several group lenses, cluster lenses and late-type galaxy lenses (e.g. Negrello et al. 2010, Bussmann et al. 2013, Negrello et al. 2014). The $\mathrm{H} \alpha$ and Hi lensing samples will be ideal to examine the diversity in the origin of the lensing optical depth to the high redshift Universe.

Having a large sample of lensing systems will make a wide range of new science applications of lensing possible. Rare lens configurations will become available to study, such as extreme high magnification events probing the morphologies of background galaxies at $\sim 100 \times$ improved angular resolution or more (conservatively excluded from our modelling in section 2), or rare "jackpot" or "double jackpot" lenses with multiple galaxies at different redshifts aligned along the line of sight (e.g. Gavazzi et al. 2008). The latter systems can yield very useful constraints on dark matter density profiles, by providing two or more lines of sight past the closest galaxy. They were also originally envisaged as a probe of cosmological parameters, but a generalisation of the mass-sheet degeneracy has been discovered (Schneider 2014) that will limit this application, unless additional modelling assumptions are invoked. Further statistical constraints on halo density profiles will come from the number counts and redshift distributions of lenses and sources (e.g. Short et al. 2012), and from the interface of strong and weak gravitational lensing (e.g. Gavazzi et al. 2007). Image flux ratio anomalies have also been recognised as being caused by halo substructure (e.g. Dalal \& Kochanek 2002, Vegetti et al. 2012), so large samples of lens system will directly constrain semi-analytic models, particularly using the $S K A$ lensing samples that are immune to the effect of dust obscuration in the foreground lenses. Large lens samples will also make it possible to investigate the stellar initial mass function as a function of galaxy types (e.g. Spiniello et al. 2011, Dutton et al. 2013).

The background sources will at best only be marginally resolved by their discovery observations with Euclid, WFIRST or SKA, but they will be among the brightest line emitters on the sky, so will be easily amenable to follow-up observations. The high angular resolution of $30-40 \mathrm{~m}$-class telescopes will be very well suited to high surface brightness arcs. The $\mathrm{H} \alpha$-emitters will be unchallenging targets in $\mathrm{H} \alpha$ for the Thirty Metre Telescope (TMT) or the European Extremely Large Telescope (E-ELT), and a 1-hour exposure with e.g. the METIS instrument on E-ELT will be sufficient to detect and resolve $\mathrm{Pa} \alpha$ in the $\mathrm{H} \alpha$-selected sample. Scaling from local ultraluminous star-forming galaxies (Veilleux, Kim 
\& Sanders 1999, Farrah et al. 2013), many $\mathrm{H} \alpha$-selected lensed galaxies will be easily detectable in far-infrared lines with the proposed SPICA space observatory (e.g. Roelfsema et al. 2012). Diagnostic line ratios may be subject to differential magnification effects (e.g. Serjeant 2012), but this may be corrected for in single systems or statistically in the population through source and lens modelling. Alternatively, differential magnification may be indirectly addressed through a comparison of emission line profiles with those of spatially resolved lines (e.g. Omont et al. 2013).

\section{CONCLUSIONS}

Euclid will be able to generate a sample of $\sim 10^{3}$ strong gravitational lens events with known background redshifts and $\sim 100 \%$ reliability by selecting $>12 L_{*} \mathrm{H} \alpha$ emitting galaxies at redshifts $z<1.7$; the $S K A$ will similarly be able to compile up to $\sim 10^{5}$ lensing events with $\sim 100 \%$ reliability and known source redshifts by selecting $>12 M_{*}$ systems in an Hi survey at $z<2$.

The author thanks the anonymous referee for helpful suggestions, the Science and Technology Facilities Council for support under grant ST/J001597/1, and many colleagues in the Euclid Strong Lensing and Galaxy Evolution working groups for stimulating discussions.

Facilities: Euclid, SKA.

\section{REFERENCES}

Abdalla, F.B., Blake, C., Rawlings, S., 2010, MNRAS, 401, 743 Auger, M.W., et al., 2009, ApJ, 705, 1099

Baugh, C.M., Lacey, C.G., Frenk, C.S., Benson, A.J., Cole, S., Granato, G.L., Silva, L., Bressan, A., 2004, New A. Rev., 48, 1239

Blain, A.W., 1996, MNRAS, 283, 1340

Bussmann, R.S., et al., 2013, ApJ, 779, 25

Calzetti, D., et al., 2010, ApJ, 714, 1256

Condon, J.J., 1992, ARA\&A, 30, 575

Dalal, N., Kochanek, C.S., 2002, ApJ, 572, 25

Dutton, A.A., Maccio, A.V., Mendel, J.T., Simard, L., 2013, MNRAS, 432, 2496

Eales, S.A., 2014, MNRAS submitted (arXiv:1312.1242)
Eales, S.A., et al., 2010, PASP, 122, 499

Eisenhardt, P.R., et al., 1996, ApJ, 461, 72

Farrah, D., et al., 2013, ApJ, 776, 38

Faure, C., et al., 2011, A\&A, 529, A72

Flores, H., Hammer, F., Elbaz, D., Cesarsky, C.J., Liang, Y.C., Fadda, D., Gruel, N., 2004, A\&A, 415, 885

Gavazzi, R., et al., 2007, ApJ, 667, 176

Gavazzi, R., et al., 2008, ApJ, 677, 1046

Gavazzi, R., Marshall, P.J., Treu, T., Sonnenfeld, A., 2014, ApJ, 785,144

Geach, J.E., et al., 2010, MNRAS, 402, 1330

González-Nuevo, J., et al., 2012, ApJ, 749, 65

Jackson, N., 2008, MNRAS, 389, 1311

Lagos, C. Del P., Baugh, C.M., Lacey, C.G., Benson, A.J.; Kim, H.-S., Power, C., 2011, MNRAS 418, 1649

Lapi, A., et al., 2011, ApJ, 742, 24

Laureijs, R., et al., 2011, arXiv:1110.3193

Lima, M., Jain, B., Devlin, M., 2010, MNRAS, 406, 2352

Navarro, J.F., Frenk, C.S., White, S.D.M., 1997, ApJ, 490, 493

Negrello, M., et al., 2010, Science, 330, 800

Negrello, M., et al., 2014, MNRAS, 440, 1999

Omont, A., et al., 2013, A\&A, 551, A115

Perrotta, F., Baccigalupi, C., Bartelmann, M., De Zotti, G., Granato, G.L., 2002, MNRAS, 329, 445

Perrotta, F., Magliocchetti, M., Baccigalupi, C., Bartelmann, M., De Zotti, G., Silva, L., Danese, L., 2003, MNRAS, 338, 623

Roelfsema, P., et al., 2012, SPIE, 8442, 0R

Schneider, P., 2014, preprint, arXiv:1406.6152

Sedgwick, C., et al., 2013, MNRAS, 436, 395

Serjeant, S., et al., 1995, MNRAS, 276, L31

Serjeant, S., Gruppioni, C., Oliver, S., 2002, MNRAS, 330, 621

Serjeant, S., 2012, MNRAS, 424, 2429

Sheth, R.K., Tormen, G., 1999, MNRAS, 308, 119

Short, J., et al., 2012, arXiv:1206.4919

Spergel, D., et al., 2013, preprint, arXiv:1305.5425

Spiniello, C., Koopmans, L.V.E., Trager, S.C., Czoske, O., Treu, T., 2011, MNRAS, 417, 3000

Swinbank, A.M., Smail, I., Chapman, S.C., Blain, A.W., Ivison,

R.J., Keel, W.C., 2004, ApJ, 617, 64

Swinbank, A.M., et al., 2010, Nature, 464, 733

Treu, T., 2010, ARA\&A, 48, 87

Vegetti, S., et al., 2012, Natur, 481, 341

Veilleux, S., Kim, D.-C., Sanders, D.B., 1999, ApJ, 522, 113

Viera, J.D., et al., 2013, Nature, 495, 344

Wang, Y., Turner, E.L., 1996, ApJ, 464, 114

Wuyts, S., et al., 2013, ApJ, 779, 135

Zwaan, M.A., et al., 2003, AJ, 125, 2842 